\title{
Patterns and outcome of surgical management of goitres at Bugando Medical Centre in northwestern Tanzania
}

\author{
PHILLIPO L. CHALYA ${ }^{1 *}$, PETER RAMBAU ${ }^{2}$, JOSEPH B. MABULA ${ }^{1}$, EMMANUEL S. KANUMBA ${ }^{1}$, \\ GODFREY GIITI ${ }^{1}$ ALPHONCE B. CHANDIKA ${ }^{1}$ and JAPHET M. GILYOMA ${ }^{1}$ \\ ${ }^{1}$ Department of Surgery, Weill-Bugando University College of Health Sciences, Mwanza, Tanzania \\ ${ }^{2}$ Department of Pathology, Weill-Bugando University College of Health Sciences, Mwanza, Tanzania
}

\begin{abstract}
Despite the well established endemicity of goitres little work has been done on the management of goiters in Tanzania. A cross-sectional study was conducted at Bugando Medical Centre (BMC) in Mwanza, Tanzania to determine the pattern and outcome of surgical management of goitres. Data was collected using a pre-tested, coded questionnaire. A total of 152 patients were studied of which $140(92.1 \%)$ were females and males were 12 (7.9\%) (F: $\mathrm{M}=11.7: 1)$. Their ages ranged from 18 to 72 years (mean $=38.4 \pm 12.5$ years). The mean duration of illness was 9.2 years. The thyroid gland size at admission was grade III in $63.8 \%$ of patients. Multinodular goitres were reported in $51.3 \%$ of patients. The majority of patients $(92.1 \%)$ presented with euthyroid goitres and the remaining $(7.9 \%)$ patients had toxic goitres. Pressure symptoms and cosmetic disfigurement were the common indications for thyroidectomy in $47.4 \%$ and $23.7 \%$, respectively. Near total thyroidectomy and total thyroidectomy were the surgical procedures performed for benign and malignant goitres in $47.3 \%$ and $8.1 \%$ of patients, respectively. Simple multinodular goitres were the most common histopathological pattern accounting for $67.2 \%$ of cases. Twelve $(7.9 \%)$ patients had a histologically proven thyroid malignancy, of which follicular and papillary carcinoma were reported in $41.7 \%$ and $33.3 \%$ of cases, respectively. Post-operative complications rate was $7.9 \%$. The mean length of hospital stay was 14.4 days (range 3 to 34 days). Five patients died giving a mortality rate of $3.4 \%$. In conclusion, this study has shown that the pattern of surgical goitres seen at Bugando Medical Centre is similar to what is reported from other parts of the world. However, the majority of patients present for surgery very late with huge goitres predisposing them to increased risk of post-operative complications, prolonged length of hospital stay and cost of medical care. It is therefore recommended that health education should be given to the community about the cause, prevention and treatment options so that patients could seek early medical attention.
\end{abstract}

Key words: goiters, pattern, outcome, surgical management, Tanzania

\section{Introduction}

Goitre, defined as enlargement of the thyroid gland that normally weighs $25-30 \mathrm{~g}$, is a common endocrine disease that is reported to affect approximately $4-15 \%$ of a given population worldwide (Tumbridge et al., 1977; Matesa et al., 2002; Takayo et al., 2002). Goitres are a common endocrine disease in Tanzania after diabetes mellitus and are a single commonest cause of surgical admissions to Bugando Medical Centre (Bugando Medical Centre- Medical Record Database 2008-2009). The etiological spectrum of thyroid diseases ranges from a variety of thyroid tumors, altered functional states and inflammatory conditions, to a rare form of disorders (Takayo et al., 2002; Matesa et al., 2002). Ultrasonographic results and autopsy findings indicate that goitre could be present in as much as $30 \%$ and $50 \%$ of the general population respectively without clinical manifestation (Takayo et al., 2002). Apart from being cosmetically disfiguring, a huge thyroid swelling can

\footnotetext{
${ }^{*}$ Correspondenceo: Dr. Phillipo Leo Chalya; E-mail: drphillipoleo@yahoo.com
} 
compress adjacent structures such as oesophagus, recurrent laryngeal nerve, superior vena cava and trachea. Compression of the trachea may seriously compromise the patency of the airway and lead to threatening respiratory obstruction (Gardiner \& Russell, 1995).

Thyroid surgery has been reported to be the mainstay of the treatment of surgical goitres and is considered as a safe procedure in well equipped settings with suitable experience to anticipate and avoid the occurrence of possible surgical complications (Mattioli et al., 1996; Bakheit et al., 2008; El Bushra et al., 2009). Many treatment modalities have been described for the surgical management of various forms of goitres, including lobectomy, sub-total thyroidectomy, near-total thyroidectomy and total thyroidectomy. However, the choice of surgical approach and the extent of tissue resection for the benign thyroid diseases remain controversial (Bellantone et al., 2002). In order to improve the outcome of these patients, most surgeons have been looking for a treatment that results in the lowest recurrence and complication rate (Acun et al., 2004). Subtotal thyroidectomy because of its low incidence of postoperative complications, has previously been advocated by some for the treatment of benign thyroid disease, but recurrence rates as high as $45 \%$ have been reported (Waldstrom et al., 1998; Acun et al., 2004; Salman \& Omer, 2007).

Recent studies have reported total thyroidectomy to be the gold standard treatment for thyroid cancer, multinodular goitre and graves. However, due to its associated risk of postoperative complications, most surgeons avoid the procedure for the treatment of benign thyroid diseases (Acun et al., 2004; Efremidou et al., 2009). Near-total thyroidectomy has been reported to achieve both low recurrence and complication rates when compared with the rates reported in the literature for total thyroidectomy and has shown to be an effective and safe surgical treatment option for various benign thyroid diseases. However, its longterm follow up has not been documented in literature (Acun et al., 2004; El Bushra et al., 2009). There is paucity of information in our setting regarding thyroid diseases including goitres. In this study, we report our experiences with goitres and their surgical management outcome in our local setting. The study will provide basis for improvement of treatment outcome of these patients.

\section{Materials and Methods}

\section{Study design and setting}

This was a cross sectional study which was conducted at Bugando Medical Centre (BMC), in Mwanza, Tanzania over a two-year period from February 2008 to January 2010. Bugando Medical Centre is a 1000 bed, consultant and teaching hospital for the Weill-Bugando University College of Health Sciences (WBUCHS) and other paramedics. It is one of the four consultant and tertiary referral hospitals in the Tanzania. BMC provides services to all patients from neighboring towns and those referred from peripheral hospitals in the Lake Victoria Zone.

\section{Study subjects}

The study included all patients who were admitted to the hospital for surgical treatment of goitres. Patients who refused to consent for the study were excluded from the study. All patients included in the study were pre-operatively evaluated by means of history taking and physical examination. The size of the goitres was graded from 0-III according to WHO classification system (WHO/UNICEF/ICCIDD, 1994). Wayne's clinical index (Lester, 1980) was used for clinical diagnosis of thyrotoxic goitres. Blood was taken (in patients who were 
found to have thyrotoxic symptoms) for determination of thyroid function tests (TSH, T4 and T3) by using Enzyme linked iso-sorbent assay (ELISA) method. Plain neck and chest radiographs were performed in patients suspected to have retrosternal goitres. Ultrasound and radioactive imaging of the thyroid gland were not performed routinely. Computed tomography scan of the neck and chest in patients with massive, retrosternal or clinically malignant goiter, or in a suspected posterior extension of the nodules was not performed due to its unavailability. Fine needle aspiration biopsy was performed only in selected cases. Antithyroid medications were used for patients who were found to have thyrotoxic symptoms before surgery to attain euthyroid state. Lugol's iodine was prescribed in some patients to reduce the vascularity of the gland. A preoperative laryngoscopic examination of the vocal cords was not done routinely.

All recruited patients were operated under general anesthesia with endotracheal intubations and muscle relaxation. Intra-operatively, recurrent laryngeal nerves (RLN) were routinely identified on both sides and every attempt was made to identify and preserve the parathyroid glands. Those glands with compromised blood supply were excised, diced and re-implanted in the sternocleidomastoid muscle. All wounds were closed with rubber drains left in situ. Intra-operative findings were documented by the operating surgeons. The weights of the thyroid specimens removed at operation were recorded. All postoperative thyroid tissues specimens removed surgically were subjected to histopathological examination for diagnostic confirmation. Surgeon's assessment of the patients' voice was a reliable method to suspect vocal cord palsy during the postoperative period. Laryngoscopic examination was advised postoperatively in patients with hoarseness or loss of voice quality. Postoperative serum calcium levels were selectively checked in all patients undergoing total thyroidectomy. In the absence of any complication all patients were discharged on the third day. All patients were followed until discharge or death.

\section{Data collection and analysis}

Data were collected using a pre-tested, coded questionnaire. Included in the questionnaire were socio-demographic data, clinical presentation, clinical diagnosis, indications for surgery, histopathological results, type of operation performed, post-operative complications and treatment outcome. Data collected was analyzed using SPSS computer software version 11.5.

\section{Ethical consideration}

All patients admitted to the hospital for surgical treatment of goitres were, after informed written consent, included in the study. Approval to conduct the study was sought from the WBUCHS/BMC Joint Ethic Review Committee before the commencement of the study.

\section{Results}

Between February 2008 and January 2010, a total of 152 patients who were admitted to BMC for surgical management of goitres were studied. 140 (92.1\%) were females and males were $12(7.9 \%)$ with a female to male ratio of 11.7: 1 . Their ages ranged from 18 to 72 years with a mean and median of 38.4 years and 36.8 years, respectively. The peak age incidence was 3140 years age group. The majority of patients, 96 (63.2\%) were younger than 40 years. The majority of patients, $99(65.1 \%)$ came from the rural areas located a considerable distance 
from Mwanza City and most of them, 82 (53.9\%) had either primary or no formal education. The majority of patients $(86.8 \%)$ presented with a long standing goitres (Table 1 ).

Table 1: Clinical presentation of patients with goitres

\begin{tabular}{lll}
\hline Clinical presentation & Frequency & Percentage \\
\hline Duration of goitre (in years) & & \\
$<1$ & 20 & 13.2 \\
$1-5$ & 97 & 63.8 \\
$>5$ & 35 & 23.0 \\
Reasons for medical consultation & & \\
Large neck swelling & 92 & 60.5 \\
Pressure symptoms & 32 & 21.1 \\
Thyrotoxic symptoms & 12 & 7.9 \\
Rapid growing swelling & 10 & 6.6 \\
Neck pain & 4 & 2.6 \\
Abscess formation & 2 & 1.3 \\
Thyroid gland size / grade & & \\
I & 8 & 5.3 \\
II & 42 & 27.6 \\
III & 102 & 67.1 \\
Lobe involved & & \\
All lobes & 86 & 56.6 \\
Right lobe & 23 & 15.1 \\
Left lobe & 21 & 13.8 \\
Solitary nodule & 22 & 14.5 \\
\hline
\end{tabular}

The duration of illness ranged from 1 month to 15 years with a mean of 9.2 years. The majority of patients $(63.8 \%)$ presented between one and five years of onset of illness. The reasons for seeking medical treatment are shown in table 2. The thyroid gland size at admission was grade III in $67.1 \%$ of patients. All lobes were enlarged in $56.6 \%$ of the cases (Table 2). The majority of patients, 140 (92.1\%) presented with euthyroid goitres and the remaining $12(7.9 \%)$ patients presented with toxic goitres which were all treated with antithyroid drugs before surgery with good response. No patient presented with hypothyroid goitres. Multinodular goitres were the most common pre-operative clinical diagnosis made (Table 2).

Table 2: Clinical diagnosis

\begin{tabular}{lll}
\hline Clinical diagnosis & Frequency & Percentage \\
\hline Multinodular goitres & 78 & 51.3 \\
Solitary thyroid nodules & 20 & 13.2 \\
Simple diffuse goitres & 20 & 13.2 \\
Neoplastic goitre & 18 & 11.8 \\
Toxic goitres & 12 & 7.9 \\
Thyroid abscess & 2 & 1.3 \\
Retrosternal goitre & 2 & 1.3 \\
\hline
\end{tabular}

Pressure symptoms (47.4\%) and cosmetic disfigurement $(23.7 \%)$ were the most common indications for surgery. Other indications included suspicious malignancy $(11.8 \%)$, toxic goiter $(7.9 \%)$, recurrent goiter $(7.9 \%)$ and thyroid abscess $(1.3 \%)$. Thyroid surgery was 
performed in 148 (97.4\%) patients. Four patients had advanced inoperable thyroid cancer (confirmed by histopathological examination of Incisional thyroid biopsy) for which only supportive therapy was provided. Near-total thyroidectomy was the most common type of thyroid surgery performed in $(47.3 \%)$ patients (Table 3$)$. Tracheostomy was performed in two patients, one due to tracheomalacia in a huge longstanding multinodular goitre and another after total thyroidectomy for thyroid cancer. Twelve $(7.9 \%)$ patients underwent secondary thyroidectomy for recurrence goitres; some of them had their initial surgery (primary thyroidectomy) decades ago. The duration of surgery ranged from 20 minutes to 160 minutes with a mean of 68.5 minutes and the mean weight of thyroid tissue removed was 452 grams (Range $=30-1600$ grams).

Table 3: Surgical procedure performed $(\mathrm{N}=148)$

\begin{tabular}{lcc}
\hline Surgical procedure performed & Frequency & Percentages \\
\hline Near-total thyroidectomy & 70 & 47.3 \\
Sub-total thyroidectomy & 24 & 16.2 \\
Nodulectomy & 20 & 13.5 \\
Lobectomy + isthmusectomy & 18 & 12.2 \\
Total thyroidectomy \pm lymph node dissection & 12 & 8.1 \\
Incision \& drainage (thyroid abscess) & 2 & 1.4 \\
\hline
\end{tabular}

Simple goiters accounted for over three quarters of the types of goiters. Twelve patients $(7.9 \%)$ had a histopathologically proven thyroid malignancy, of which follicular carcinoma was the most common type in $41.7 \%$ of cases (Table 4 ).

Table 4: Histopathological (results) diagnosis $(\mathrm{N}=152)$

\begin{tabular}{lcc}
\hline Histopathological results & Frequency & Percentage \\
\hline Simple goitres & $\mathbf{( 1 1 6 )}$ & $\mathbf{( 7 6 . 3 )}$ \\
Multinodular goitres & 78 & 67.2 \\
Simple colloid goitres & 14 & 12.1 \\
Diffuse hyperplastic goitres & 13 & 11.2 \\
Solitary nodular goitres & 11 & 9.5 \\
Toxic goitres & $\mathbf{( 1 2 )}$ & $\mathbf{( 7 . 9 )}$ \\
Diffuse toxic goitres & 7 & 58.3 \\
Toxic nodular goitre & 5 & 41.7 \\
Thyroid adenomas & $\mathbf{( 1 0 )}$ & $\mathbf{( 6 . 6 )}$ \\
Papillary adenoma & 5 & 50.0 \\
Follicular adenoma & 5 & 50.0 \\
Thyroid carcinomas & $\mathbf{( 1 2 )}$ & $\mathbf{( 7 . 9 )}$ \\
Follicular carcinoma & 5 & 41.7 \\
Papillary carcinoma & 4 & 33.3 \\
Mixed papillary-follicular carcinoma & 2 & 16.7 \\
Hurthle cell carcinoma & 1 & 8.3 \\
Thyroiditis & $\mathbf{( 2 )}$ & $\mathbf{( 1 . 3 )}$ \\
Pyogenic & 1 & 50.0 \\
Tuberculous & 1 & 50.0 \\
\hline
\end{tabular}

Twelve $(7.9 \%)$ patients developed post-operative complications. Significant intra-operative hemorrhage requiring post-operative blood transfusion $(33.3 \%)$ was the most common complication following thyroidectomy. Other post-operative complications included 
hoarseness of voice (3), wound sepsis (1), aspiration pneumonia (1), transient tetany (1) and abscess formation requiring incision and drainage (1).

The overall length of hospital stay ranged from 3 to 34 days with a mean of 14.4 days. Five patients died giving a mortality rate of $3.4 \%$. Two patients who had thyroid abscess were also HIV positive, died of HIV related complications. One patient who had tracheostomy due to tracheomalacia died of sudden tracheostomy tube obstruction six hours after operation. The other two patients died of advanced thyroid cancer

\section{Discussion}

The pattern of surgical goitres seen at Bugando Medical Centre in Tanzania appears to be similar to what is reported from other parts of the world (Mellese \& Taddese, 2001; Hill et al., 2004; Bekele et al., 2004; Abede \& Osman, 2006; El-Bushra et al., 2009). The age distribution of goitres in our study is comparable with other studies (Bekele et al., 2004; Abede \& Osman, 2006) but the female to male ratio of $11.7: 1$ in this study is higher than that reported elsewhere (Abede \& Osman, 2006; El-Bushra et al., 2009). These differences in sex ratios can be explained by an increased occurrence of goitre in both sexes due to high goitre prevalence in a given population. The sex differences in the tendency to visit health facilities when sick may also hide the true sex predominance. Therefore, this might not reflect true sex preponderance in the community. The actual sex ratio should be obtained in a broader community based study.

Most of the patients in our study came from the rural areas located a considerable distance from Mwanza City. Similar observation was also reported in other African studies (Hill et al., 2004; Bekele et al., 2004; Abede \& Osman, 2006). This observation may explain the reason for late presentation to hospital in the majority of cases. Delayed presentation for treatment is still a common feature in most patients in Africa, as reported by other studies (Hill et al., 2004; Bekele et al., 2004; Abede \& Osman, 2006; El-Bushra et al., 2009). In the present study, the majority of patients presented with a long standing goitres. The absence of symptoms besides the bulky mass might be the most probable reason for this. The considerable distance of the hospital from the goitre endemic areas, the slow growth of the mass and its presence in most of the inhabitants in the region might also contribute to the delay in presentation. Hospital visits are also found to be determined by cosmetic interest, educational status, recent onset of new symptoms, and psychosocial trauma and stigmatization the patients suffered because of their illness (Bekele et al., 2004).

The results of this study show that simple multinodular goitres was the most common type of goitres seen at Bugando Medical Centre. A similar pattern has also been reported by other studies (Mellese \& Taddese, 2001; Hill et al., 2004; Bekele et al., 2004; Abede \& Osman, 2006; El-Bushra et al., 2009). Simple multinodular goiter is the most common type of goitre in endemic iodine-deficiency regions where there is very low iodine content in the water and food. This leads to formation of reduced levels of thyroid hormones and hence the goitre. The high prevalence of simple multinodular goitres in these areas reflects low level of iodine in food and in water in these regions.

The rate of thyroid carcinoma in our study is higher than those reported elsewhere in Africa (Soyannwo et al., 1995; Makuria, 1977; Mellese \& Taddese, 2001). However, other studies have reported even high rates (Bekele et al., 2004; Abede \& Osman, 2006). The age of occurrence and histological pattern of these malignancies in this study is similar to other reports in Africa (Bekele et al., 2004; Abede \& Osman, 2006). In this study, follicular 
carcinoma was the commonest type of thyroid cancer and tended to affect the young age group. This finding is in agreement with other studies done in Africa (Bekele et al., 2004; Abede \& Osman, 2006). Chow et al. (2001) in China and Franssila et al. (1981) in Switzerland have reported high rates of papillary carcinoma. The relatively younger age of occurrence of follicular malignancy may be related to endemicity of goitres in the country. Follicular carcinoma is said to follow long-standing iodine deficiency goitres and its prevalence is more common in areas of endemic goiters (William et al, 1977, Bekele et al., 2004). Further studies are needed to explain the high rate of thyroid cancer in this region.

Thyroid abscess is infrequently mentioned in literatures and there are few clinical reports. Bekele et al. (2004) in Ethiopia reported seven cases with frank thyroid abscess that were managed with simple abscess drainage; of these, one patient was seropositive for HIV. In our study, thyroid abscess was found in two patients who were also HIV positive (data not shown). An underlying immunosuppression and super infection of cystic goitre and introduction of organisms during tattooing might be responsible for its development in our patients.

In our study, pressure symptoms and cosmetic disfigurement were the most common indications for surgery. This finding is similar to studies done elsewhere (Abede \& Osman, 2006; Salman \& Omer, 2007; El-Bushra et al, 2009). Acun et al (2004) in Greece reported toxic symptoms as the most common indication for thyroidectomy. The indication for surgery is determined by the prevalence of goitre in a certain locality.

Although thyroidectomy has been reported to be a well established surgical procedure and the main stay of treatment of goitres (Giddings, 1998), the approach and the extent of tissue resection for benign goitres remain controversial (Bellantone et al., 2002). Many surgeons advocate a surgical procedure that results into low complications and recurrence rates (Acun et al., 2004). In our study, a near-total thyroidectomy was the most common type of operation performed particularly in patients with toxic and large multinodular goitres and was found to be effective and relatively safe within the current hospital setup. The type of thyroid surgery done in our institution was found to be similar with reports from other centers (Acun et al., 2004; El-Bushra et al., 2009). However, a long term follow up study is required to assess the long term outcome of this type of surgery. Some studies advocate total thyroidectomy for the treatment of benign thyroid diseases due to its low rates of recurrence (Perzik \& Katz, 1967; Jacob et al., 1983; Karlan et al., 1984). However, such radical surgery needs meticulous surgical procedure to preserve the parathyroid glands, recurrent laryngeal nerves and to avoid bleeding. Total thyroidectomy also requires meticulous post-operative care with intensive care facilities which are not always available in developing countries and hence near-total thyroidectomy is often a justifiable procedure for benign goitres. The provision of more surgical training and intensive post thyroidectomy care facilities must precede the adoption of more radical surgery for benign lesions, as has been recommended worldwide (El-Bushra et al., 2009).

The post-operative complication rate in the present study is in agreement with other studies reported elsewhere (Bekele et al., 2004; Abede \& Osman, 2006; El-Bushra et al., 2009). In our study, hemorrhage sometimes requiring blood transfusion was the most common immediate complication which is comparable with other studies (Abede \& Osman, 2006; ElBushra et al., 2009). This is due to the presence of huge goitres with highly vascularized thyroid tissue in most of our patients and the presence of advanced thyroid malignancies. We also noted that the duration of surgery, post operative stay and postoperative complications was higher in patients operated for huge goitres and for thyroid carcinoma. 
The mean duration of hospital stay in our study was about two weeks, which is higher than that reported in other studies ((Abede \& Osman, 2006; El-Bushra et al., 2009). The reason for prolonged mean hospital stay in our study is attributable to long preoperative stay due to long operation waiting list and time required for patient preparation. The occurrences of post operative complications are also responsible for post operative longer stay. Social reasons such as long distance and lack of money to pay for the hospital bills and for transport back home also contribute to prolonged post operative hospital stay. The overall mortality rate in our study was found to be higher than that reported in studies done elsewhere (Bekele et al., 2004, El-Bushra et al., 2009). Higher mortality rate in our study may be attributable to the presence of post-operative complications, HIV infections presenting with thyroid abscess and advanced thyroid malignancies.

Despite lack of diagnostic facilities such as thyroid ultrasound, radioactive imaging and CT scan, the study has highlighted our experiences with goitres and their outcome of surgical management in our limited-resource environment. In conclusion, this study has shown that the pattern of surgical goitres seen at Bugando Medical Centre appears to be similar to what is reported from other parts of the world. The majority of patients present for surgery very late with huge goitres predisposing them to increased risk of post-operative complications, prolonged length of hospital stay and cost of medical care. Thyroidectomy is one of the most common surgical procedures done at BMC and has been performed with low post-operative complication and mortality rates. It is therefore recommended that health education should be given to the community not only about the cause and prevention but also about the treatment options so that patients could seek medical attention earlier than shown in the study. The importance of follow up clinics should also be emphasized.

\section{Acknowledgements}

To all nurses in the surgical wards/ outpatient clinics and operating theatres, physicians and surgeons and all those who were involved in the care of these patients are highly acknowledged.

\section{References}

Abede, B. \& Osman, M. (2006) Goitre in a teaching hospital in the north western Ethiopia. East and Central African Journal of Surgery11, 21-27.

Acun, Z., Comert, M., Cihan, A., Ulukent, S.C., Ucan, B. \& Cakmak, G.K. (2004) Near-total thyroidectomy could be the best treatment for thyroid disease in endemic regions. Archives of Surgery139, 444-447.

Bakheit, M.A., Mahadi, S.I. \& Ahmed, M.E. (2008) Indications and outcome of thyroid gland surgery in Khartoum Teaching Hospital. Khartoum Medical Journal 1, 34-37.

Bekele, A., Tamrat, G., Osman, M., Sentayehu, T. \& Sissay, B. (2004) Patterns of surgical thyroid disease and operative treatment in Gondar College of Medical Sciences, north western Ethiopia. East and Central African Journal of Surgery 9, 87-93.

Bellantone, R., Lombardi, C.P. \& Bossola, M. (2002) Total thyroidectomy for management of benign thyroid disease: review of 526 cases. World Journal of Surgery 26, 1468-1471.

Chow, T.L., Chu, W., Lim, B.H. \& Kwok, S.P. (2001) Outcomes and complications of thyroid surgery: retrospective study. Hong Kong Medical Journal 7, 261-265.

disease. Surgery 62, 436-440. 
Efremidou, E.I., Michael, S., Papageorgiou, M.S., Liratzopoulos, N. \& Manolas, K.J. (2009) The efficacy and safety of total thyroidectomy in the management of benign thyroid disease: a review of 932 cases. Canadian Journal of Surgery 52 , 39-44.

El Bushra, A.D., Mohamed, I.M., Awadalla, M.A. \& Mohamed, Y.B. (2009) Thyroidectomy at El Obeid Hospital, Western Sudan. Khartoum Medical Journal 2, 158-161.

Franssila, K., Saen, E. \& Teppo, L. (1981) Incidence of different morphological types of thyroid cancers in Nordic countries. Acta Pathologica Microbilogica Immunologica Scandinavica 89, 49-52.

Gardiner, K.R. \& Russell, C.F.J. (1995) Thyroidectomy for large multinodular goiter. Journal of the Royal College of Surgeons of Edinburgh 40, 367-370.

Giddings, A.E. (1998) The history of thyroidectomy. Journal of the Royal Society of Medicine 91, 3-6.

Hill, A.G., Mwangi, I. \& Wagana, L. (2004) Thyroid disease in a rural Kenya hospital. East African Medical Journal 81, 631-633.

Jacob, J., Aland, J. \& Ballinger, J. (1983) Total thyroidectomy: a review of 213 patients. Annals of Surgery 197, 542-549.

Karlan, M., Katz, B., Dunkelman, D., Uyeda, R. \& Gleischman, S. (1984) A safe technique for

Lester, F.T. \& Tsega, E. (1980) Thyrotoxicosis and primary myxedema in 13 Ethiopian patients. Ethiopian Medical Journal 18, 15-21.

Makuria, T. (1972) Surgical goiter in Ethiopia. Ethiopian Medical Journal 15, 169-173.

Matesa, N., Tabain, I., Dabelić, N., Petric, V. \& Kusić, Z. (2002) Diagnostic relevance of fine needle aspiration cytology for follicular lesions of the thyroid: Retrospective study. Croatian Medical Journal 43, 606-609.

Mattioli, F.P., Torre, G.C., Borgonovo, G., Arezzo, A., Amato, A. \& De Negri, A. (1996) Surgical treatment of multinodular goiter. Annali Italini Di Chirurgia 67, 341-345.

Mellese, G. \& Taddese, B. (2001) Changes in the patterns of surgical thyroid diseases in Zewditu hospital, Addis Ababa. Ethiopian Medical Journal 41, 179-184.

Neck Surgery 6, 1014-1021.

Perzik, S.L. \& Katz, B. (1967) The place of total thyroidectomy in the management of thyroid

Salman, Y.G. \& Omer, A.E. (2007) Total thyroidectomy for Bilateral Benign Thyroid Diseases: Safety profile and therapeutic Efficacy. Kuwait Medical Journal 39, 149-152.

Soyannwo, O.A., Ajao, O.G., Agbejule, O.A. \& Amanor-Boadu, S.D. (1995) Anaesthesia and surgical aspects of thyroid swelling: the Ibadan experience. East African Medical Journal 72, 675-77.

Takayo, K., Humiaki S., Takahiro, S., Michio, A. \& Shinya, K. (2002) Management of Nodular goiters and their operative indications. Surgery Today 30, 722-726.

thyroidectomy with complete nerve dissection and parathyroid preservation. Head

Tumbridge, W.M.G., Evered, D.C. \& Hall, R. (1977) The spectrum of thyroid diseases in a community: The Whickham study. Endocrinology 7, 481-93.

Waldstrom, C., Zedenius, J., Guinea, A., Reeve, T. \& Delbridge, L. (1998) Multinodular goiter presenting as a clinical single nodule: how effective is hemithyroidectomy?. Australian and New Zealand Journal of Surgery 69, 34-36.

WHO/UNICEF/ICCIDD. (1994) Indicators for assessing iodine deficiency disorders and their control through salt iodination. Geneva: WHO.

William, E.D., Doniach, I., Biarnason, O. \& Michie W. (1977) Thyroid cancers in iodine rich areas, A histopathological study. Cancer 39, 2 15-21 8 